\title{
Effect of graphene oxide nanosheets on the physico-mechanical properties of chitosan/bacterial cellulose nanofibrous composites
}

\author{
Amir Azarniya $^{\mathrm{a}}$, Niloofar Eslahi ${ }^{\mathrm{a}}$, Nafiseh Mahmoudi ${ }^{\mathrm{a}}$, Abdolreza Simchi $^{\mathrm{a}, \mathrm{b}^{*}}$ \\ ${ }^{\text {a } D e p a r t m e n t ~ o f ~ M a t e r i a l s ~ S c i e n c e ~ a n d ~ E n g i n e e r i n g, ~ S h a r i f ~ U n i v e r s i t y ~ o f ~ T e c h n o l o g y, ~ A z a d i ~ A v e n u e, ~ P . O . ~}$ \\ Box 11365-9466, Tehran, Iran \\ ${ }^{\mathrm{b}}$ Institute for Nanoscience and Nanotechnology, Sharif University of Technology, Azadi Avenue, P.O. Box \\ 11365-9466, Tehran, Iran
}

*Corresponding author: Tel.: +98 216616 5261; Fax: +98 216600 5717; simchi@sharif.edu (A. Simchi) 


\begin{abstract}
In this work, novel chitosan/bacterial cellulose $(\mathrm{CS} / \mathrm{BC})$ nanofibrous composites reinforced with graphene oxide (GO) nanosheets are introduced. As cell attachment and permeability of nanofibrous membranes highly depend on their fiber diameter, the working window for successful electrospinning to attain sound nanofibrous composites with a minimum fiber diameter was determined by using the response surface methodology. It is shown that the addition of GO nanosheets to $\mathrm{CS} / \mathrm{BC}$ significantly reduces the average size of the polymeric fibers. Their mechanical properties are also influenced and can be tailored by the concentration of GO. Fourier transform infrared spectroscopy reveals hydrogen bonding between the GO nanosheets and the polymer matrix. A decrease in the hydrophilicity of the electrospun nanofibers and their water vapor permeability with the addition of GO are also reported. The prepared nanofibrous composites are potentially suitable candidates for biomedical applications such as skin tissue engineering and wound dressing.
\end{abstract}

Keywords: A. Biocomposite; A. Nano-structures; B. Mechanical properties; E. Electrospinning

\title{
1. Introduction
}

Over the past decade, a miscellaneous assortment of nanofibrous biocomposites have been developed with the aim of generating an alternative or complementary approach to prepare scaffold for tissue engineering, wound dressing, and drug delivery [1]. Porosity, morphology, mechanical properties and rate of chemical degradation of nanofibrous membranes must meet the demands of end application [2]. Owing to their unique characteristics such as large surface-to-volume ratio, flexibility in surface functionalities, superior mechanical performance, high level of porosity and permeability, nanofibers can be functionalized for numerous biomedical applications $[1,3]$. Several methods have been developed to synthesize biodegradable and biocompatible nanofibrous scaffolds such as self-assembly, phase separation, interfacial polymerization, rapidly initiated polymerization, template- or pattern-assisted growth, vapor-liquid-solid growth, hydrothermal synthesis, electrospinning, island in the sea and drawing $[4,5]$. There is currently an increasing interest in production of biopolymeric nanofibers by electrospinning, though the process is a facile and efficient technique to fabricate various polymers and polymer-based composites [6]. Among a wide range of 
natural or synthetic biopolymers, chitosan (CS) and its derivatives have attracted a great interest for biomedical applications. This is because of their advantageous characteristics such as biocompatibility, biodegradability, non-toxicity, antibacterial and hemostatic activity [7]. Chitosan is widely utilized for the preparation of nanofibrous biocomposites as antimicrobial wound-dressing material [8]. Since the fabrication of CS nanofibers by electrospinning is challenging $[9,10]$, additives such as collagen, poly(vinyl alcohol), poly(caprolactone), poly(vinylpyrrolidone), poly(ethylene oxide) and poly(ethylene terephthalate) are utilized [7]. Recently, the addition of bacterial cellulose (BC) to CS to prepare functional nanofibers has gained attention [9]. This nontoxic polysaccharide is synthesized by a certain bacteria and regarded as an essential ingredient for several recognized products used in wound dressing [11]. Bacterial cellulose provides important characteristics for biomedical applications including hydrophilicity, biocompatibility, biodegradability, transparency, high crystallinity, stability at a wide range of temperatures and good mechanical properties with elastic modulus up to $114 \mathrm{GPa}$ [12-15]. Whiskers of $\mathrm{BC}$ obtained by the acid hydrolysis of bacterial cellulose microfibrils were imbedded in electrospun PEO nanofibers [16] and ethylene vinyl alcohol (EVOH) fibers [17] to enhance mechanical properties. Electrospun BC nanofibers were reinforced by incorporated multiwalled carbon nanotubes [18]. Importantly, there is also an structural similarity between $\mathrm{CS}$ and $\mathrm{BC}$, bringing composite materials of both the physicochemical properties into existence [9]. However, the mechanical properties of $\mathrm{CS} / \mathrm{BC}$ nanofibrous composites may not be compatible with the natural skin tissue; thereby, it seems necessary to strengthen the fibrous mats using hydrophilic, biocompatible and chemically stable reinforcements.

Graphene oxide (GO)-reinforced polymer-matrix composites have recently attracted significant interest for biomedical applications [19]. Graphene oxide, a new carbon two-dimensional nanomaterial is chemically functionalized derivative of graphene nanosheets with hydroxyl and epoxide groups on the basal plane and carboxyl groups at the edges. These polar functional groups not only give birth to hydrophilicity and good dispersion of GO in water and some other polar solvents, but also enables it to form strong interactions such as hydrogen bonding with various polymer matrix. Li et al. [20] showed that addition of GO to CS fibers prepared by wet spinning enhanced the mechanical properties. Electrospun poly(vinyl alcohol)/CS/GO e nanofibers (123-200 nm) with enhanced mechanical strength were prepared by Liu et al. [21]. Lee et al. [22] 
showed that the growth and differentiation of stem cells were enhanced by using GO sheets as cell culture platforms. Mazaheri et al. [23] prepared flexible GO-CS composite layers using solvent casting method and showed that the GO addition made a dramatic improvement to stem cell proliferation and antibacterial activity as well as mechanical properties compared with a pristine chitosan film.

Fabrication of a nanofibrous composite scaffold with physico-mechanical characteristics as good as natural skin tissue is one of the key issues in tissue engineering. The aim of this research is to fabricate GOreinforced $\mathrm{CS} / \mathrm{BC}$ fibrous nanocomposites with enhanced mechanical properties. The modified strength is attained by tailoring the fiber diameter and the concentration of GO. It should be noted that the fiber diameter not only affects the mechanical properties of nanofibrous membranes but also influences cell adhesion and cell growth kinetics [24]. Therefore, it is important to attain long and continuous nanofibers with a high uniformity. In electrospinning, the diameter of fibers is controlled by solution parameters (concentration, viscosity, conductivity and surface tension), process parameters (applied voltage, collection distance and flow rate), and ambient parameters (humidity and temperature) [25]. Obviously, there is some degree of interactions between these parameters; hence, it is necessary to determine the effect of main parameters on the fiber diameter. Response surface methodology (RSM) is one of the statistical optimization techniques with an ability to optimize two or more effective parameters simultaneously [26]. Recently, Dhurai et al. [27] have employed RSM to optimize the electrospinning process for curcumin-loaded chitosan/poly (lactic acid) nanofibers. In this work, we employed RSM to prepare CS/BC nanofibers with variable diameters. The effect of applied voltage, needle to collector distance and the $\mathrm{CS} / \mathrm{BC}$ ratio on the fiber diameter was investigated.

Afterwards, GO nanosheets were added to the polymeric suspension to prepare biocompatible nanocomposites. Effects of GO addition on the fiber diameter, mechanical properties, permeability and hydrophilicity of the resulting composites were studied.

\section{Experimental procedure}

\subsection{Materials preparation}

Chitosan (medium molecular weight, 190-310 kDa, 75\% deacetylated), poly(ethylene oxide) (PEO) with a molecular weight of $900 \mathrm{kDa}$ and acetic acid (AA technical, 90\%) were purchased from Sigma-Aldrich. 
Bacterial cellulose (a gel with concentration of $3 \mathrm{wt} \%$ nanofibrillated $\mathrm{BC}$ in water) was provided from Nano Novin Polymer Co., Iran. 3\% (w/v) chitosan solution in aqueous AA solution and 5\% (w/v) BC suspension were prepared and mixed at different volume ratios of 1:1, 4.5:1 and 8:1 under magnetic stirring for $24 \mathrm{~h}$ at room temperature. To achieve nanofibers of high uniformity, a PEO solution $(5 \% \mathrm{w} / \mathrm{v})$ was added to the mixtures at different amounts. Natural graphite powder purchased from Johnson Matthey Co. (London, UK) was utilized to synthesize GO nanosheets according to the modified Hummers method [28]. The GO suspension was obtained through dispersion of a certain amount of synthesized GO into distilled water and sonicated overnight at room temperature.

\subsection{Electrospinning of polymer fibers}

The polymer solutions were electrospun via a single jet electrospinning apparatus (Model HVPS-35/500, ANSTCO, Iran). The polymer solution was fed into the horizontally aligned syringe with a needle orifice of $0.55 \mathrm{~mm}$ in inner diameter. An aluminum foil was utilized for covering grounded collector to collect the produced nanofibers. The injection rate was $0.3 \mathrm{~mL} / \mathrm{h}$. A needle to collector distance of $120 \mathrm{~mm}$ and an applied voltage of $20 \mathrm{kV}$ were used to electrospun the $\mathrm{CS} / \mathrm{BC}$ blends containing different amounts of PEO solution ( $(0,3,6$ and 10 vol.\%.). The addition of PEO was aimed to increase spinnability of the CS solution through reducing its viscosity [29]. Finally, the electrospun fibers were dried overnight in a desiccator at room temperature. The rate of fiber deposition was determined by measuring the areal density of the fiber mats (the mass of electrospun fibers per unit area, $\mathrm{g} / \mathrm{cm}^{2}$ ) after spinning for 10, 30, 60, 120, 180 and $250 \mathrm{~min}$.

\subsection{Optimization of electrospinning parameters by RSM}

Optimization of the electrospinning processing parameters was performed by employing the response surface methodology (RSM). Primary tests showed that the applied voltage, electrospinning distance, and the CS/BC ratio could vary in the range of 16 to $22 \mathrm{kV}, 100$ to $140 \mathrm{~mm}$, and 1 to 8 , respectively. The designed levels for the experimental factors are summarized in Table 1. The generated experiment conditions designed by a BoxBehnken approach are listed in Table 2. Quantitative relationship between the parameters and fiber diameter was also modeled using a quadratic polynomial function. The experiments were run at random to randomize distribution of varying factors. 


\subsection{Electrospinning of GO-containing solutions}

The GO suspension $(5 \mathrm{~g} / \mathrm{L})$ was added to the $\mathrm{CS} / \mathrm{BC}$ suspension (volume ratio of 4.65$)$ at different concentrations of $0,0.5,1,1.5$ and $2 \mathrm{wt} \%$. The mixtures were magnetically stirred for $24 \mathrm{~h}$ and then electrospun at an applied voltage of $22 \mathrm{kV}$ and spinning distance of $100 \mathrm{~mm}$.

\subsection{Materials Characterization}

The size and morphology of fibrous structures were examined using field-emission scanning electron microscope (FE-SEM, Philips Model XL30, Netherland) at an accelerating voltage of $30 \mathrm{kV}$. Before analysis, nanofibers were sputter-coated with gold for $60 \mathrm{~s}$. The mean diameter of the nanofibers was obtained for each sample by measuring the diameter of 50 random fibers chosen from their FE-SEM images by employing an image analysis software (Image J, National Institute of Health, Bethesda, MD). To study molecular interactions and chemical structure of GO-containing CS/BC nanofibers, Fourier transform infrared spectroscopy (FT-IR, Varian 1000 FT-IR Scimitar series, PIKE technologies, USA) was employed in transmission mode in the wavenumber range of $4000-400 \mathrm{~cm}^{-1}$ at a resolution of $1 \mathrm{~cm}^{-1}$. A water contactangle goniometry (JC2000C, Zhongcen, Shanghai, China) was employed to evaluate the effect of GO addition on the wettability of the nanofibrous membranes after a residence time of $5 \mathrm{~s}$ at room temperature. The mechanical properties of the mats were determined by a universal tensile testing machine (AG-5000G, Shimadzu, Japan) according to ASTM Standard D882. The cross-section and the gauge length were in $20 \times 5$ $\mathrm{mm}^{2}$ and $20 \mathrm{~mm}$ sizes, respectively. A crosshead speed of $5.0 \mathrm{~mm} / \mathrm{min}$ and a load cell of $100 \mathrm{~N}$ were employed. The water vapor permeability (WVP) of the fibrous membranes was determined using a Desiccant Method according to ASTM Standard E96. Specimens were sealed to the open mouth of a glass beaker containing silica gel as a desiccant, and the assembly was placed in a lab dish with controlled atmosphere containing saturated solution of ammonium nitrate salt. The temperature and relative humidity was fixed during the test at $20^{\circ} \mathrm{C}$ and $65 \%$, respectively. Periodic weighting determined the rate of water vapor transmission through the polymeric membranes into the silica gel. The value of WVP was calculated using the following equation:

$W V P=\frac{G T}{t A . \Delta P}$ 
where $G$ is the weight change due to vapor transmission, $T$ thickness, $t$ time, $A$ test area, and $\Delta \mathrm{P}$ vapor pressure difference between two specific surfaces.

\section{Results}

\subsection{Electrospinning of chitosan/bacterial cellulous fibers}

Fig. 1 shows FE-SEM images of electrospun CS/BC (1:1) blends containing different amounts of PEO. Without PEO, tiny droplets in the range of 50-800 nm were formed (Fig. 1a). The addition of 3\% (w/v) PEO solution resulted in the formation of short and thin fibers bridging between two or more droplets (Fig. 1b). At higher concentrations, the electrospinning capacity of the polymeric solution increased with a decrease in the mean diameter of the fibers and the size of the interconnected voids (Fig. 1c and 1d). We have found that suspensions containing 10\% PEO are more spinnable and yield more uniformity in the fiber diameter. As shown in Fig. 1e, this sample has long and continuous nanofibers with a high uniformity. To optimize the electrospinning condition, the effect of processing parameters on the mean fiber diameter was investigated. Fig. 2 shows typical FE-SEM images of the prepared CS/BC nanofibrous along with their corresponding size distribution plots. The mean diameter of the continuous nanofibers, as determined by randomly measuring of 50 fibers, is shown in Table 2. We have experimentally found that with increasing the $\mathrm{CS} / \mathrm{BC}$ ratio up to 4.5 , the size uniformity increased but at higher ratios the uniformity was decreased. At a constant $\mathrm{CS} / \mathrm{BC}$ ratio of 4.5, a decrease in the electrospinning distance reduced the mean fiber diameter, particularly at low and high levels of the applied voltage. The effect of needle to collector distance on the fiber diameter can be described based on the amount of electric field applied on the tip of the syringe [30], which changes the jet velocity. It was also found that the mean fiber diameter slightly changed by the applied voltage. In order to determine the interactions between different variables, i.e. applied voltage $(A)$, electrospinning distance $(B)$ and $\mathrm{CS} / \mathrm{BC}$ volume ratio $(C)$, the experimental results were fitted to a polynomial model and a quadratic equation was suggested in terms of codded values as follows:

Diameter $=195-13.25 A+22.12 B-13.63 C-10.75 A B+0.75 A C-16.5 B C-24 A^{2}-2.25 B^{2}+$ $10.25 C^{2}$ 
Results of analysis of variance (ANOVA) for the proposed regression model are reported in Table 3 . The significance of each coefficient was checked using $F$-test and its associated probability. $P$-values less than 0.05 indicate that the model terms are significant. Based on this criterion, $A, B, C, B C, A^{2}$ are found to be significant model terms. The $R$-square value of 0.9721 signifies that the model is of high reliability. The $F$ value of 11.6 implies the precision of the model and a high signal to noise ratio. After removing the insignificant coefficients, the model was simplified as:

Diameter $=201.4-13.25 A+22.12 B-13.63 C-16.5 B C-26.4 A^{2}$

The ANOVA results for this reduced model is given in Table 4. A comparison between the original and adjusted models reveals an enhancement in model $F$ ratio and adequate precision (13.15) due to the model adjustment. $P$-values of all of the present terms in the reduced model are less than 0.05 as a sign of their significance. The smaller $P$-value implies that the corresponding coefficient is more significant. Based on this analysis, it was concluded that the electrospinning distance had the highest effect on the fiber diameter among the others. The surface and counter plots for the variables are shown in Fig. 3. A linear relationship between the diameter and the distance was observed in Fig. 3a. On the other hand, parameters $A$ (voltage) and $C$ (CS/BC ratio) showed an opposite effect because of their negative coefficients. The effect of CS/BC ratio $(C)$ was more significant than that of voltage $(A)$. The main reason for this finding is due to the increased number of protonated amino groups in the acidic media by increasing the chitosan content in the suspension. Thus, the higher surface charge density and conductivity of the jet form finer fibers under the applied electrical field. Fig. 4 demonstrates the interaction plot and the normal probability plot of residuals ( the sample residual divided by the square root of its estimated variance [31]). Normal residuals match a straight line (Fig. 4a), supporting an acceptable level of normality assumption. Interaction plot between electrospinning distance and $\mathrm{CS} / \mathrm{BC}$ ratio at voltage of $19 \mathrm{kV}$ is illustrated in Fig. $4 \mathrm{~b}$. The results indicated an opposite effect of spinning distance and $\mathrm{CS} / \mathrm{BC}$ ratio on the fiber diameter. In order to determine the optimum condition for attaining the finest fibers, numerical optimization was performed by the Design Expert software. The most suitable condition was attained at spinning distance of $100 \mathrm{~mm}$, applied voltage of $22 \mathrm{kV}$, and CS/BC ratio of 4.65. To validate the suggested model for prediction of fiber diameter, a confirmation experiment was conducted under 
the predicted optimum condition. The validation test yielded a mean fiber diameter of $143 \mathrm{~nm}$, which was in a good agreement with the predicted value (Table 2), supporting the accuracy of the proposed model.

\subsection{Electrospinning of GO-containing nanocomposite membranes}

Fig. 5 shows FE-SEM images of electrospun CS/BC membranes containing different contents of GO nanosheets (a: $0, \mathrm{~b}: 0.5, \mathrm{c}: 1, \mathrm{~d}: 1.5$, e and $\mathrm{f}: 2 \%$ ). The fibrous mats exhibit a bimodal distribution of fiber diameter and spider-wave-like nets, which are comprised of interlinked thin and thick fibers. The effect of GO addition on the mean fiber diameter is shown in Fig. 6a. A mat with fine fiber diameter and uniform morphology is attained at $1.5 \% \mathrm{GO}$ concentration. Although the specimen containing $2 \% \mathrm{GO}$ has the smallest fiber diameter, the mat shows a heterogeneous morphology with a film-like structure at different regions of the microstructure (Fig. 5f). A typical deposition rate for the 1.5\%GO nanocomposite is shown in Fig. 6b. The areal density increases linearly with time indicating constancy of the electrospinning rate. The rate of fiber deposition was measured as the slope of the plot for the sample containing $1.5 \% \mathrm{GO}$ and found to be $6.5 \times$ $10^{-4} \mathrm{~g} / \mathrm{cm}^{2} \min$.

To examine possible interactions between the constituents, FTIR spectrum of $\mathrm{BC}, \mathrm{CS} / \mathrm{BC}$ and $\mathrm{CS} / \mathrm{BC} / \mathrm{GO}$ fibrous membranes were recorded (Fig. 6c). All specimens exhibit a broad absorption band in the range of $3500-3000 \mathrm{~cm}^{-1}$ representing the stretching vibration of hydroxyl groups. The changes in the intensity and location of this band in different samples could be originated from some alterations in hydrogen bonding between the polymer molecules [32]. The strong peak at around $2900 \mathrm{~cm}^{-1}$ is also typical of aliphatic C-H stretching vibrations. The FTIR spectrum of pristine BC shows distinctive absorption bands at $1638 \mathrm{~cm}^{-1}$ and $1443 \mathrm{~cm}^{-1}$ corresponding to the carbonyl groups and $\mathrm{CH}_{2}$ bending vibrations, respectively. The broad peak at about $1060 \mathrm{~cm}^{-1}$ is ascribed to $\mathrm{C}-\mathrm{O}-\mathrm{C}$ stretching vibration corresponding to glycoside linkage in $\mathrm{BC}$ [33]. Since the molecular structure of $\mathrm{BC}$ and chitosan is very similar, it is expected that these two polymers have good compatibility and miscibility [34]. The FTIR spectrum of CS/BC composite exhibits three extra peaks (relative to pristine $\mathrm{BC}$ ) at 1735,1564 , and $1343 \mathrm{~cm}^{-1}$, which are attributed to the $\mathrm{C}=\mathrm{O}$ stretching of ester carbonyl formed in BC probably by acetic acid, the $\mathrm{N}-\mathrm{H}$ bending of amide II and $\mathrm{C}-\mathrm{N}$ stretching of amide III in chitosan, respectively [34-37]. This observation implies chemical interactions between BC and CS. The 
decreased intensity of the absorption band at $1443 \mathrm{~cm}^{-1}$ may also indicate a reduction in the degree of crystallinity of cellulose [38]. After the GO addition, no major change in the locations of the main peaks is observed. The peaks at $1736 \mathrm{~cm}^{-1}$ and $1560 \mathrm{~cm}^{-1}$ are assigned to the $\mathrm{COOH}$ groups of GO and amide II N-H of CS, respectively. The peak at about $1150 \mathrm{~cm}^{-1}$ belongs to $\mathrm{C}-\mathrm{O}-\mathrm{C}$ stretching vibrations of the $\mathrm{GO}$ epoxy groups as well as the bridge oxygen stretching bands of the saccharide structure [39, 40]. Therefore, the FTIR spectrum of $\mathrm{CS} / \mathrm{BC} / \mathrm{GO}$ nanocomposite can be regarded as a combination of pristine $\mathrm{CS} / \mathrm{BC}$ nanofibers and graphene oxide with a slight shift in some of the characteristic peaks. This observation reveals intermolecular hydrogen bonding between the components, possibly between oxygen functional groups of the GO sheets, carbonyl and hydroxyl groups of bacterial cellulose, and chitosan amino groups [23].

\subsection{Effect of GO nanosheets on the properties of fibrous membranes}

Tensile properties of CS/BC membranes with and without addition of $1.5 \mathrm{wt} \% \mathrm{GO}$ nanosheets are presented in Fig. 7a. The results determine the enhancing effect of GO nanosheets on the tensile strength of the nanofibrous mats. The strength and elastic modulus increased from 25 to $35 \mathrm{MPa}$ and 340 to $730 \mathrm{MPa}$, respectively, whereas the elongation decreased from 19 to $9.5 \%$. It is suggestible that GO nanosheets reduce the chains segmental mobility and increase their entanglement [41]. As a result, the strength increases while the restricted movement of the polymer chains decreases the elongation. The effect of GO concentration on the hydrophilicity and water vapor permeability of the CS/BC membranes is shown in Fig. 7b. The results indicate that the addition of GO reduces the hydrophilicity of the composite mats, in agreement with the work of Liu et al. [21]. The water vapor permeability is also reduced by GO nanosheets; for instance, the permeability of pristine CS/BC nanofibers is $3.045 \mathrm{~g} \mathrm{~Pa}^{-1} \mathrm{~h}^{-1} \mathrm{~m}^{-1}$ which is $64 \%$ higher than that of the nanocomposite containing $1.5 \% \mathrm{GO}$ with $1.631 \mathrm{~g} \mathrm{~Pa}^{-1} \mathrm{~h}^{-1} \mathrm{~m}^{-1} \mathrm{WVP}$.

\section{Discussion}

Morphological studies revealed that, electrospinning of the $\mathrm{CS} / \mathrm{BC}$ blend (without PEO addition) yielded tiny droplets (Fig. 1a). This observation highlighted the instability of the process that led to electrospraying [42]. In order to improve spinnability of the $\mathrm{CS} / \mathrm{BC}$ solution, an easily electrospinnable polymer (PEO) was utilized. It was found that the higher amount of PEO in the $\mathrm{CS} / \mathrm{BC}$ blend facilitated the propensity of fiber 
formation (Fig. 1c and 1d) which could be attributed to the hydrogen bonding between ether groups of PEO and hydroxyl and amino groups of BC/CS that enhanced the jet stability [43]. Results also indicated that the fiber diameter was strongly influenced by processing parameters including applied voltage, electrospinning distance and $\mathrm{CS} / \mathrm{BC}$ volume ratio (Fig. 3). It was found that a decrease in the electrospinning distance at different levels of the applied voltage reduced the fiber diameter, particularly at low and high values of voltage. At a constant voltage, a decrease in the needle to collector distance resulted in an increase in the electric field applied on the tip from which the electrospinning jet was ejected. As a consequence, the jet was accelerated more quickly to the collector and finer fibers were attained. Considering the effect of applied voltage, a quadratic behavior in the response surface was noticed. It has been known that the fiber diameter is under control of some factors affected by voltage such as the amount of solution ejected from tip, jet elongation, etc. [44]; hence, the applied voltage may increase or decrease the diameter of the fibers depending on these parameters. It was also shown that the fiber diameter was reduced by utilizing a more concentrated CS solution. An increase in the chitosan content in the electrospinning suspension was accompanied by more protonated amino groups that enhanced the surface charge density and conductivity of the jet [45]. Meanwhile, the results indicated that at the low levels of electrospinning distance, the effect of $\mathrm{CS} / \mathrm{BC}$ ratio on the fiber diameter was insignificant. Nevertheless, at longer distances, there was a sharp reduction in the diameter by increasing the $\mathrm{CS} / \mathrm{BC}$ ratio as the role of protonated amino group became more important at smaller electric fields.

It was also found that the $\mathrm{GO}$ addition to the $\mathrm{CS} / \mathrm{BC}$ suspension resulted nanofibrous membranes with a bimodal size distribution and spider-wave-like morphology (Fig. 5). At high GO concentrations ( $>1.5 \%)$, a film-like structure was formed and the electrospinning became more difficult. Agglomeration of GO sheets was also noticed. It was suggested that the addition of GO nanosheets increased the viscosity of the suspension while changing its conductivity. The formation of hydrogen bonds between the polymer chains and GO was also effective. As a consequence, the presence of different molecular weight substances in the suspension led to the formation of spider-wave-like mats comprised of interlinked thin and thick fibers [46]. On the other hand, a reducing effect of the GO nanosheets on the hydrophilicity and water vapor permeability of the membranes was noticed. Finer fibers and the formation of a spider-wave-like structure should enhance 
the water contact angle due to finer structural features and changes in surface roughness [47] (see Fig. 5). The pores also became finer with more tortuosity that affected the permeability [48]. The hydrophilic nature of GO as a water absorbent agent could also be effective [49]. On the other hand, the GO addition significantly enhanced the mechanical strength in expense of some ductility loss (Fig. 7a). In polymer matrix composites, inter- and intra- molecular interactions significantly influence their mechanical properties [50]. Graphene oxide has a high degree of oxygen functional groups and because of its anionic nature, its interactions with chitosan at the molecular level can improve the mechanical strength. Here, it is important to mention that the mechanical properties of human skin strongly depend on (i) skin site on the body, (ii) the orientation of the loading direction with respect to the Langer's lines, (iii) strain rate, and (iv) skin age [51]. As a result, the mechanical strength of the natural skin significantly varies within a wide range of experimental results. Table 5 shows the values reported for human skin and compares their mechanical properties with the prepared $\mathrm{CS} / \mathrm{BC} / \mathrm{GO}$ nanocomposites. The results reveal that one can tune the mechanical properties of the GO reinforced $\mathrm{CS} / \mathrm{BC}$ fibrous nanocomposites depending on the applicability of the material either via diameter of the electrospun fibers or the GO concentration.

\section{Conclusions}

Chitosan/bacterial cellulose nanofibrous membranes were fabricated by electrospinning. The effect of electrospinning parameters including $\mathrm{CS}$ to $\mathrm{BC}$ volume ratio, applied voltage and working distance on the fiber diameter was studied. The response surface methodology was employ to determine the most suitable processing conditions to attain uniform and fine fibrous membranes. It was shown that uniform mats with an average fiber diameter of about $143 \mathrm{~nm}$ could be prepared at the spinning distance of $100 \mathrm{~mm}$, applied voltage of $22 \mathrm{kV}$, and $\mathrm{CS} / \mathrm{BC}$ ratio of 4.65 . The effect of GO nanosheets on the electrospinning of the CS/BC blends was also studied. It was shown that GO nanosheets reduced the average fiber diameter. Formation of a spiderwave-like structure was also observed for the nanocomposites containing $\leq 1.5 \% \mathrm{GO}$. At the higher concentration ( $2 \% \mathrm{GO})$, however, formation of a film-like structure was observed. The mechanical properties under tensile loading, the hydrophilicity and water vapor permeability of the nanocomposites were also examined. An enhancement in the mechanical strength ( $40 \%$ and $115 \%$ promotion in the tensile strength and elastic modulus, respectively) with about $60 \%$ decrease in elongation was measured for the $1.5 \% \mathrm{GO}$ - 
reinforced nanocomposite as compared with the pristine $\mathrm{CS} / \mathrm{BC}$ nanofibers. The addition of the GO nanosheets was accompanied with a gradual decrease in the hydrophilicity of the nanofibrous membranes as well. The water vapor permeability of the CS/BC nanofibers was $3.045 \mathrm{~g} \mathrm{~Pa}^{-1} \mathrm{~h}^{-1} \mathrm{~m}^{-1}$ as compared to $1.631 \mathrm{~g}$ $\mathrm{Pa}^{-1} \mathrm{~h}^{-1} \mathrm{~m}^{-1}$ for the nanocomposite containing $1.5 \% \mathrm{GO}$.

\section{Acknowledgments}

The authors thank funding support from the Grant Program of Sharif University of Technology (No. G930305) and the Elite National Institute of Iran. They also acknowledge the contribution of Mr. Eng. Abolfazl Azarniya (Sharif University of Technology) in DOE analysis.

\section{References}

1. Arya N, Sharma P, Katti DS. Designing Nanofibrous Scaffolds for Tissue Engineering. Advanced Biomaterials: Fundamentals, Processing, and Applications. 2010:435-97.

2. Yang S, Leong KF, Du Z, Chua CK. The design of scaffolds for use in tissue engineering. Part I. Traditional factors. Tissue engineering. 2001;7(6):679-89.

3. Huang ZM, Zhang YZ, Kotaki M, Ramakrishna S. A review on polymer nanofibers by electrospinning and their applications in nanocomposites. Composites Science and Technology. 2003;63(15):2223-53.

4. Schiffman JD, Schauer CL. A Review: Electrospinning of Biopolymer Nanofibers and their Applications. Polymer Reviews. 2008;48(2):317-52.

5. Nguyen LTH, Chen S, Elumalai NK, Prabhakaran MP, Zong Y, Vijila C, Allakhverdiev SI, Ramakrishna S. Biological, Chemical, and Electronic Applications of Nanofibers. Macromolecular Materials and Engineering. 2013;298(8):822-67.

6. Pham QP, Sharma U, Mikos AG. Electrospinning of polymeric nanofibers for tissue engineering applications: a review. Tissue engineering. 2006;12(5):1197-211.

7. Ignatova M, Manolova N, Rashkov I. Electrospun antibacterial chitosan-based fibers. Macromolecular bioscience. 2013;13(7):860-72.

8. Abdelgawad AM, Hudson SM, Rojas OJ. Antimicrobial wound dressing nanofiber mats from multicomponent (chitosan/silver-NPs/polyvinyl alcohol) systems. Carbohydrate polymers. 2014;100:166-78. 
9. Fernandes SCM, Freire CSR, Silvestre AJD, Pascoal Neto C, Gandini A. Novel materials based on chitosan and cellulose. Polymer International. 2011;60(6):875-82..

10. Homayoni H, Ravandi SAH, Valizadeh M. Electrospinning of chitosan nanofibers: Processing optimization. Carbohydrate polymers. 2009;77(3):656-61.

11. Abeer MM, Mohd Amin MC, Martin C. A review of bacterial cellulose-based drug delivery systems: their biochemistry, current approaches and future prospects. The Journal of pharmacy and pharmacology. 2014;66(8):1047-61.

12. Hsieh YC, Yano H, Nogi M, Eichhorn SJ. An estimation of the Young's modulus of bacterial cellulose filaments. Cellulose. 2008;15(4):507-13.

13. Fu L, Zhang J, Yang G. Present status and applications of bacterial cellulose-based materials for skin tissue repair. Carbohydrate polymers. 2013;92(2):1432-42.

14. Czaja W, Romanovicz D, Malcolm Brown R. Structural investigations of microbial cellulose produced in stationary and agitated culture. Cellulose. 2004;11(3-4):403-11.

15. Bodin A, Backdahl H, Fink H, Gustafsson L, Risberg B, Gatenholm P. Influence of cultivation conditions on mechanical and morphological properties of bacterial cellulose tubes. Biotechnology and bioengineering. 2007;97(2):425-34.

16. Park WI, Kang M, Kim HS, Jin HJ. Electrospinning of poly (ethylene oxide) with bacterial cellulose whiskers. Macromolecular Symposia: Wiley Online Library; 2007;249(1):289-94.

17. Martínez-Sanz M, Olsson RT, Lopez-Rubio A, Lagaron JM. Development of bacterial cellulose nanowhiskers reinforced EVOH composites by electrospinning. Journal of Applied Polymer Science. 2012;124(2):1398-408.

18. Chen P, Yun YS, Bak H, Cho SY, Jin HJ. Multiwalled carbon nanotubes-embedded electrospun bacterial cellulose nanofibers. Molecular Crystals and Liquid Crystals. 2010;519(1):169-78.

19. Yoon OJ, Jung CY, Sohn IY, Kim HJ, Hong B, Jhon MS, Lee NE. Nanocomposite nanofibers of poly (d, 1-lactic-co-glycolic acid) and graphene oxide nanosheets. Composites Part A: Applied Science and Manufacturing. 2011;42(12):1978-84. 
20. Li Y, Sun J, Du Q, Zhang L, Yang X, Wu S, Xia Y, Wang Z, Xia L, Cao A. Mechanical and dye adsorption properties of graphene oxide/chitosan composite fibers prepared by wet spinning. Carbohydrate polymers. 2014;102:755-61.

21. Liu Y, Park M, Shin HK, Pant B, Choi J, Park YW, Lee JY, Park SJ, Kim HY. Facile preparation and characterization of poly(vinyl alcohol)/chitosan/graphene oxide biocomposite nanofibers. Journal of Industrial and Engineering Chemistry. 2014;20(6):4415-20.

22. Lee WC, Lim CHY, Shi H, Tang LA, Wang Y, Lim CT, Loh KP. Origin of enhanced stem cell growth and differentiation on graphene and graphene oxide. ACS nano. 2011;5(9):7334-41.

23. Mazaheri M, Akhavan O, Simchi A. Flexible bactericidal graphene oxide-chitosan layers for stem cell proliferation. Applied Surface Science. 2014;301:456-62.

24. Chen M, Patra PK, Warner SB, Bhowmick S. Optimization of electrospinning process parameters for tissue engineering scaffolds. Biophysical Reviews and Letters. 2006;1(02):153-78.

25. Chronakis IS. Novel nanocomposites and nanoceramics based on polymer nanofibers using electrospinning process-A review. Journal of Materials Processing Technology. 2005;167(2-3):283-93.

26. Khuri AI, Mukhopadhyay S. Response surface methodology. Wiley Interdisciplinary Reviews: Computational Statistics. 2010;2(2):128-49.

27. Dhurai B, Saraswathy N, Maheswaran R, Sethupathi P, Vanitha P, Vigneshwaran S, Rameshbabu V. Electrospinning of curcumin loaded chitosan/poly (lactic acid) nanofilm and evaluation of its medicinal characteristics. Frontiers of Materials Science. 2013;7(4):350-61.

28. Song P, Zhang X, Sun M, Cui X, Lin Y. Synthesis of graphene nanosheets via oxalic acid-induced chemical reduction of exfoliated graphite oxide. RSC Advances. 2012;2(3):1168.

29. Bhattarai N, Edmondson D, Veiseh O, Matsen FA, Zhang M. Electrospun chitosan-based nanofibers and their cellular compatibility. Biomaterials. 2005;26(31):6176-84.

30. Deitzel J, Kleinmeyer J, Harris D, Tan NB. The effect of processing variables on the morphology of electrospun nanofibers and textiles. Polymer. 2001;42(1):261-72.

31. Eslahi N, Dadashian F, Nejad NH. Optimization of enzymatic hydrolysis of wool fibers for nanoparticles production using response surface methodology. Advanced Powder Technology. 2013;24(1):416-26. 
32. Zhang S, Luo J. Preparation and properties of bacterial cellulose/alginate blend bio-fibers. Journal of Engineered Fibers and Fabrics. 2011;6(3):69-72.

33. Pandey M, Abeer MM, Amin MCIM, Cairul M. Dissolution Study Of Bacterial Cellulose (Nata De Coco) From Local Food Industry: Solubility Behavior \& Structural Changes. International Journal of Pharmacy and Pharmaceutical Sciences. 2014;6(6):89-93.

34. Kim J, Cai Z, Lee HS, Choi GS, Lee DH, Jo C. Preparation and characterization of a bacterial cellulose/chitosan composite for potential biomedical application. Journal of Polymer Research. 2011;18(4):739-44.

35. Lin WC, Lien CC, Yeh HJ, Yu CM, Hsu Sh. Bacterial cellulose and bacterial cellulose-chitosan membranes for wound dressing applications. Carbohydrate polymers. 2013;94(1):603-11.

36. Phisalaphong M, Jatupaiboon N. Biosynthesis and characterization of bacteria cellulose-chitosan film. Carbohydrate polymers. 2008;74(3):482-8.

37. Ionita M, Vasile E, Crica LE, Voicu SI, Pandele AM, Dinescu S, Predoiu L, Galateanu B, Hermenean A, Costache M. Synthesis, characterization and in vitro studies of polysulfone/graphene oxide composite membranes. Composites Part B: Engineering. 2014;72:108-115.

38. Bertocchi C, Delneri D, Signore S, Weng Z, Bruschi CV. Characterization of microbial cellulose from a high-producing mutagenized Acetobacter pasteurianus strain. Biochimica et Biophysica Acta (BBA)-General Subjects. 1997;1336(2):211-7.

39. Shanmugharaj A, Yoon J, Yang W, Ryu SH. Synthesis, characterization, and surface wettability properties of amine functionalized graphene oxide films with varying amine chain lengths. Journal of colloid and interface science. 2013;401:148-54.

40. Han D, Yan L, Chen W, Li W. Preparation of chitosan/graphene oxide composite film with enhanced mechanical strength in the wet state. Carbohydrate polymers. 2011;83(2):653-8.

41. Lim HN, Huang NM, Loo C. Facile preparation of graphene-based chitosan films: Enhanced thermal, mechanical and antibacterial properties. Journal of Non-Crystalline Solids. 2012;358(3):525-30.

42. Zheng J, He A, Li J, Xu J, Han CC. Studies on the controlled morphology and wettability of polystyrene surfaces by electrospinning or electrospraying. Polymer. 2006;47(20):7095-102. 
43. Pakravan M, Heuzey M-C, Ajji A. A fundamental study of chitosan/PEO electrospinning. Polymer. 2011;52(21):4813-24.

44. Tan SH, Inai R, Kotaki M, Ramakrishna S. Systematic parameter study for ultra-fine fiber fabrication via electrospinning process. Polymer. 2005;46(16):6128-34.

45. Kriegel C, Kit K, McClements DJ, Weiss J. Electrospinning of chitosan-poly (ethylene oxide) blend nanofibers in the presence of micellar surfactant solutions. Polymer. 2009;50(1):189-200.

46. Pant HR, Nam KT, Oh HJ, Panthi G, Kim HD, Kim BI, Kim HY. Effect of polymer molecular weight on the fiber morphology of electrospun mats. Journal of colloid and interface science. 2011;364(1):107-11.

47. Kim J, Kim HS, Park CH. Contribution of surface energy and roughness to the wettability of polyamide 6 and polypropylene film in the plasma-induced process. Textile Research Journal. 2015;86(5):461-471.

48. Li S, de Wijn JR, Li J, Layrolle P, de Groot K. Macroporous biphasic calcium phosphate scaffold with high permeability/porosity ratio. Tissue Engineering. 2003;9(3):535-48.

49. Zahedi P, Rezaeian I, Ranaei-Siadat SO, Jafari SH, Supaphol P. A review on wound dressings with an emphasis on electrospun nanofibrous polymeric bandages. Polymers for Advanced Technologies. 2010;21(2):77-95.

50. Eastwood E, Viswanathan S, O'brien C, Kumar D, Dadmun M. Methods to improve the properties of polymer mixtures: optimizing intermolecular interactions and compatibilization. Polymer. 2005;46(12):395770.

51. Gallagher A, Annaidh AN, Bruyère K. Dynamic tensile properties of human skin. In: IRCOBI Conference. Dublin, September, 2012. p.12-14.

52. Gilchrist MD, Annaidh AN, Destrade M. Characterising the anisotropic mechanical properties of excised human skin. Journal Of The Mechanical Behavior Of Biomedical Materials. 2012;5:139-148.

53. Silver FH, Freeman JW, DeVore D. Viscoelastic properties of human skin and processed dermis. Skin Research and Technology. 2001;7(1):18-23.

54. Jacquemoud C, Bruyere-Garnier K, Coret M. Methodology to determine failure characteristics of planar soft tissues using a dynamic tensile test. Journal of Biomechanics. 2007;40(2):468-475. 


\section{Figures caption}

Fig. 1. FE-SEM images of electrospun $\mathrm{CS} / \mathrm{BC}$ samples (1:1 volume ratio) containing different concentration of PEO solution (w/v): (a) $0 \%$, (b) 3\%, (c) 6\% and (d, e) $10 \%$.

Fig. 2. FE-SEM images and corresponding fiber diameter distribution plots for electrospun CS/BC nanofibrous.

Fig. 3. Response surface and contour plots for the fiber diameter as a function of (a) voltage and spinning distance, (b) $\mathrm{CS} / \mathrm{BC}$ ratio and voltage, and (c) $\mathrm{CS} / \mathrm{BC}$ ratio and distance.

Fig. 4 (a) Normal probability plot of residuals for the fiber diameter. (b) Interaction plot between electrospinning distance and $\mathrm{CS} / \mathrm{BC}$ ratio.

Fig.5. FE-SEM images of CS/BC nanofibers containing: (a) 0 , (b) $0.5 \%$, (c) $1 \%$, (d) $1.5 \%$, and (e, f) $2 \%$ GO.

Fig. 6. (a) Effect of GO content on the fiber diameter of the electrospun CS/BC nanocomposites. (b) Areal density versus deposition time for electrospinning of $\mathrm{CS} / \mathrm{BC}$ composite containing $1.5 \% \mathrm{GO}$ nanosheets. (c) FTIR spectra of (a) BC gel, (b) CS/BC mat, (c) CS/BC/GO nanocomposite membrane.

Fig 7. (a) Engineering stress-strain curves of CS/BC nanofibrous membranes with and without addition of $1.5 \% \mathrm{GO}$. (b) Effect of GO concentration on the water contact angle of fibrous $\mathrm{CS} / \mathrm{BC} / \mathrm{GO}$ mats. 
Figure 1
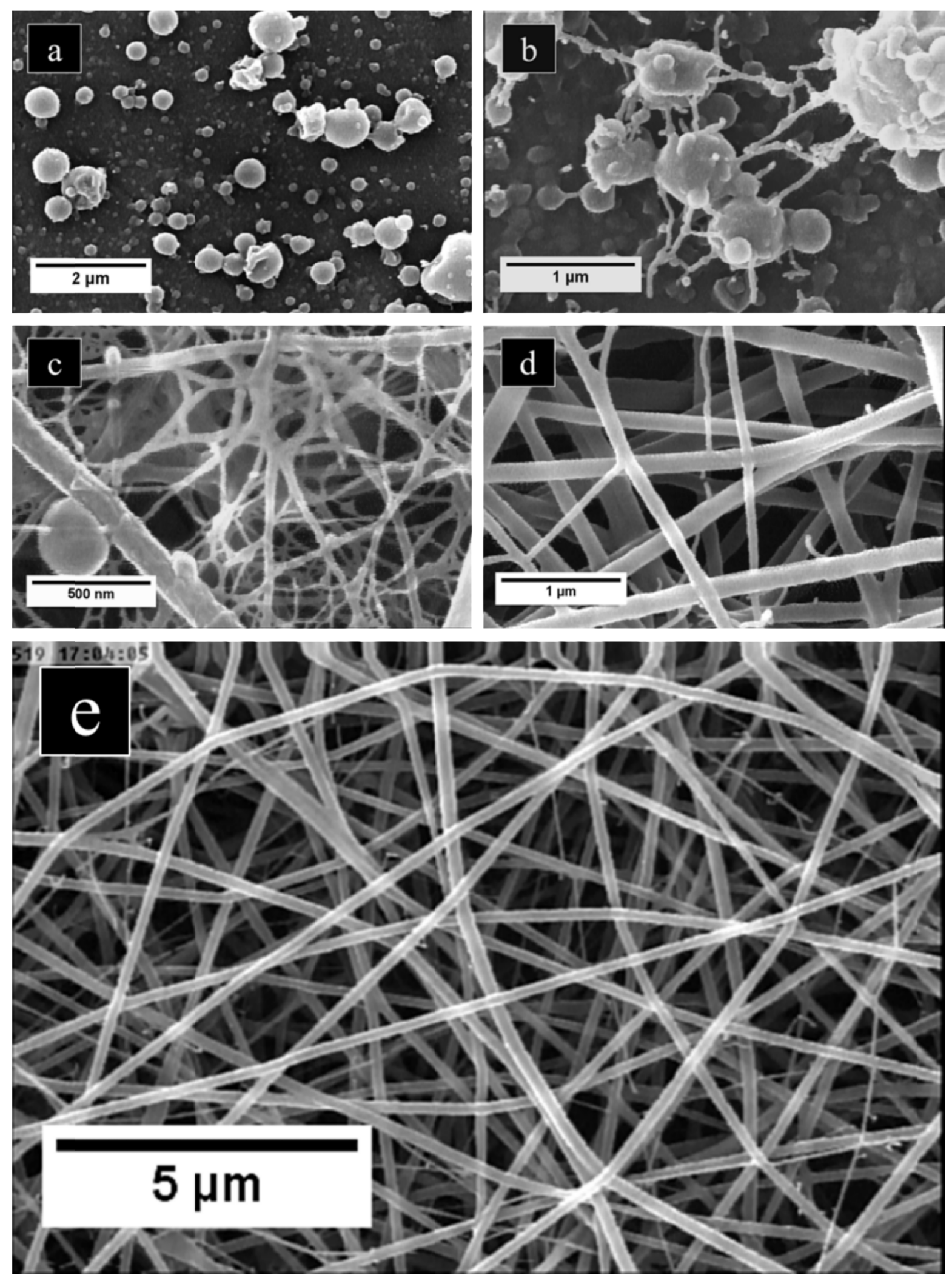
Figure 2

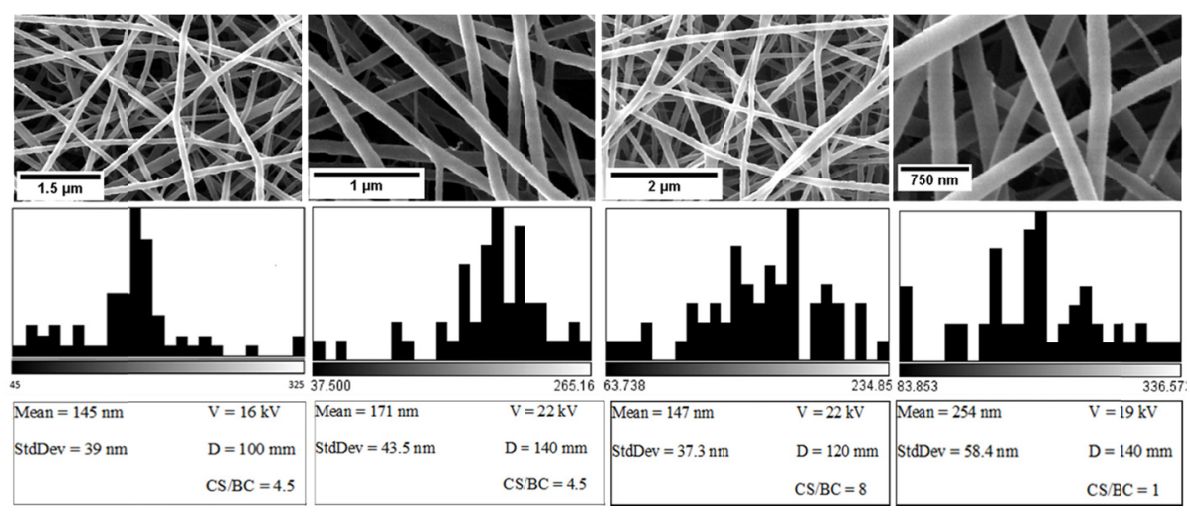




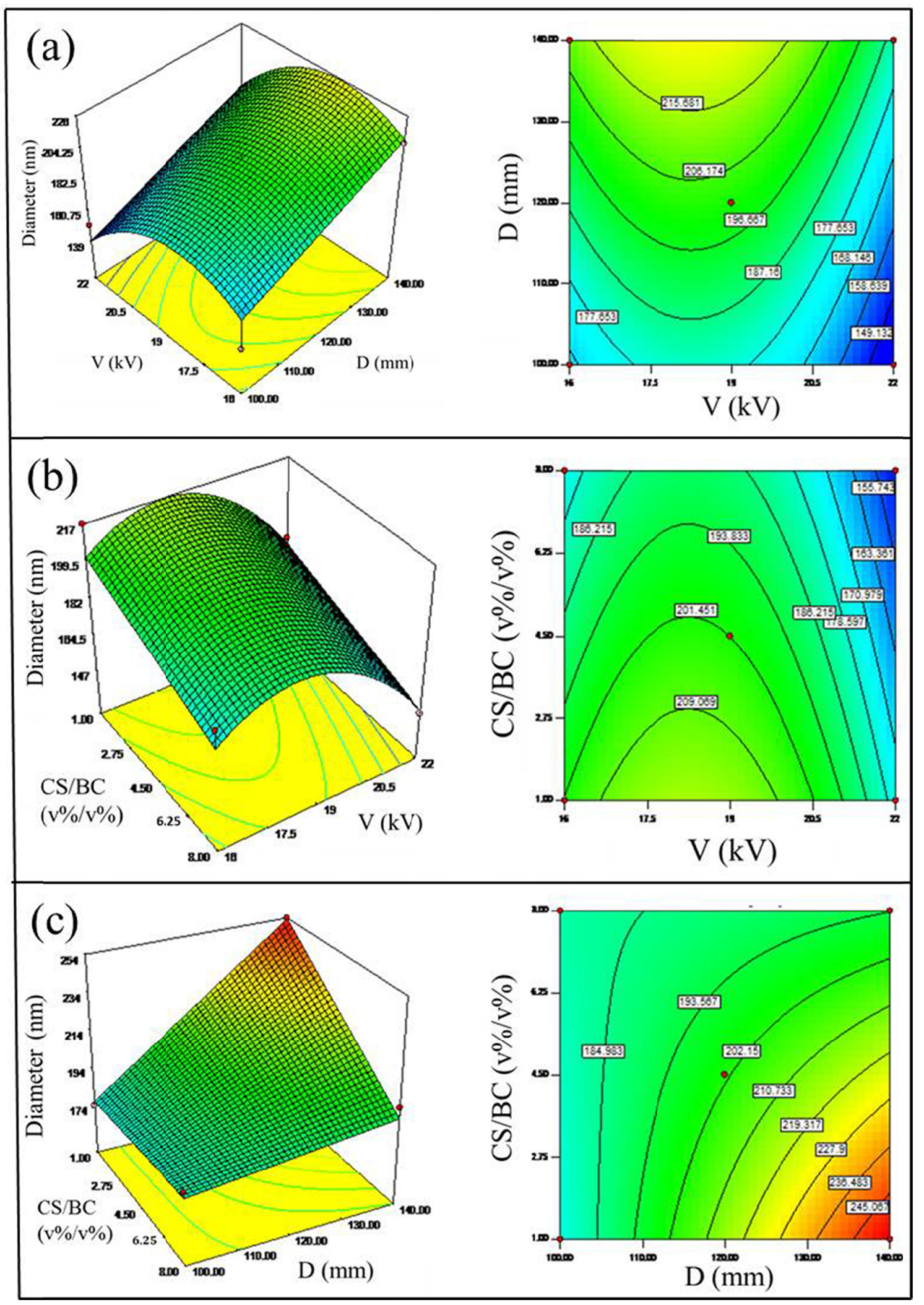




\section{Figure 4}

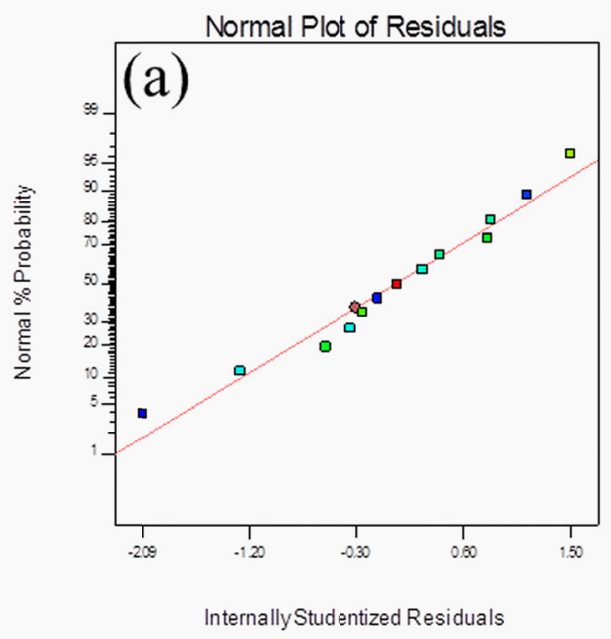

Design-Expertie Software

Diameter:(nm)

- Design Points

- C- 1.000

A $\mathrm{C}+8.000$

$\mathrm{X} 1=\mathrm{B}: \mathrm{D} /(\mathrm{mm})$

$X_{2}=C \cdot \operatorname{csiBC}(v \%)$

Actual Factor

$\mathrm{A}: \mathrm{V} /(\mathrm{KV})=19.00$

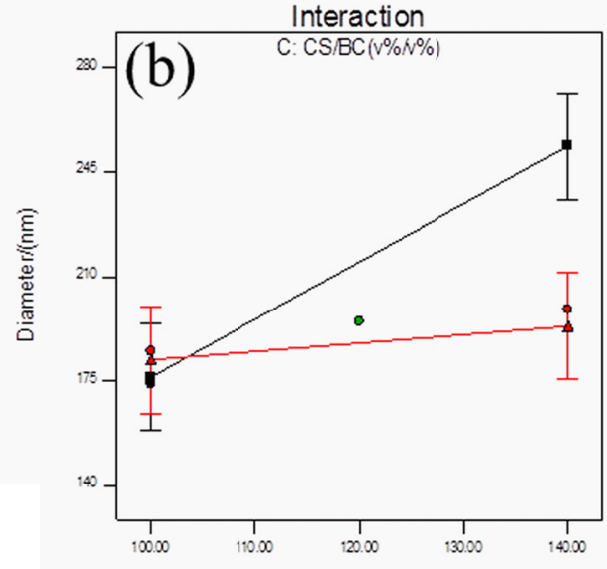

B. $D /(\mathrm{mm})$ 
Figure 5

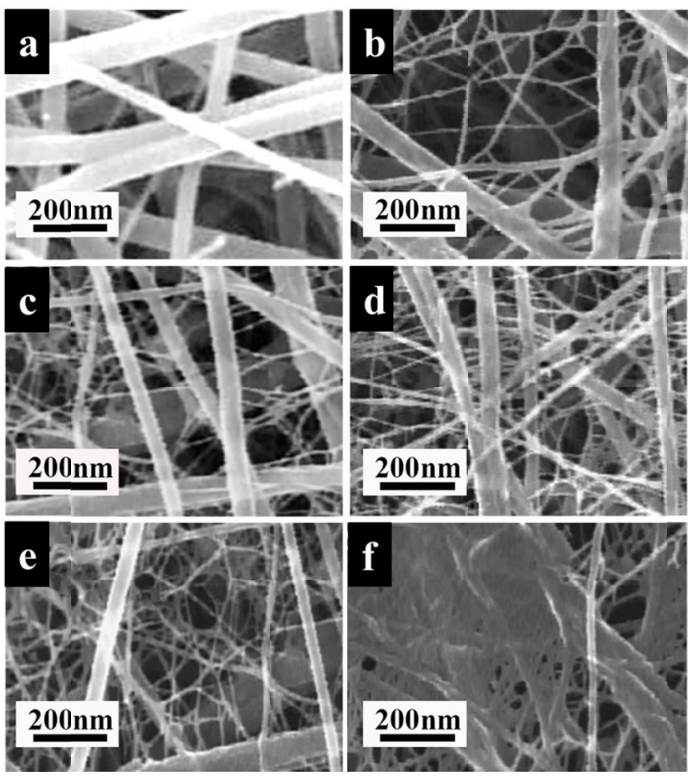




\section{Figure 6}
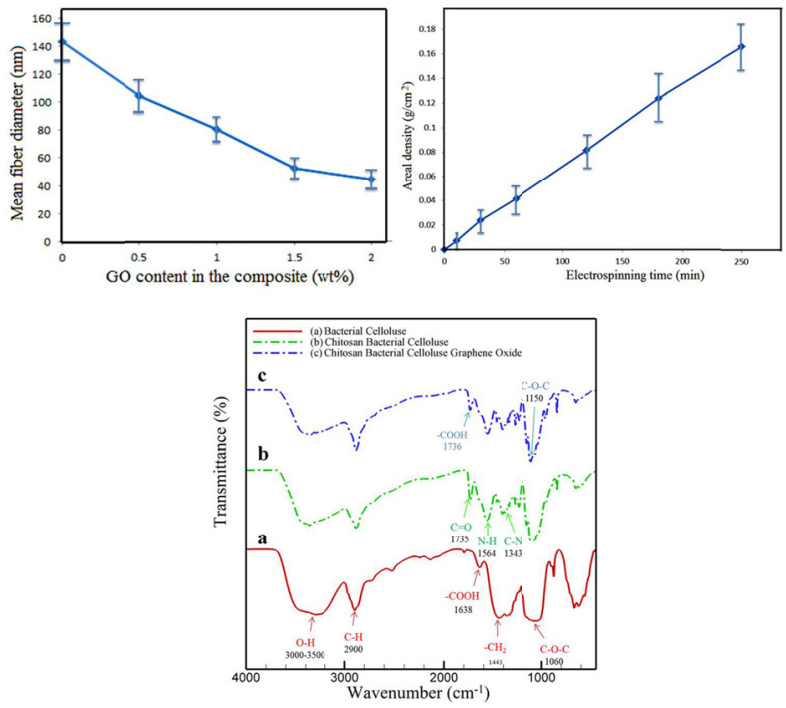
Figure 7
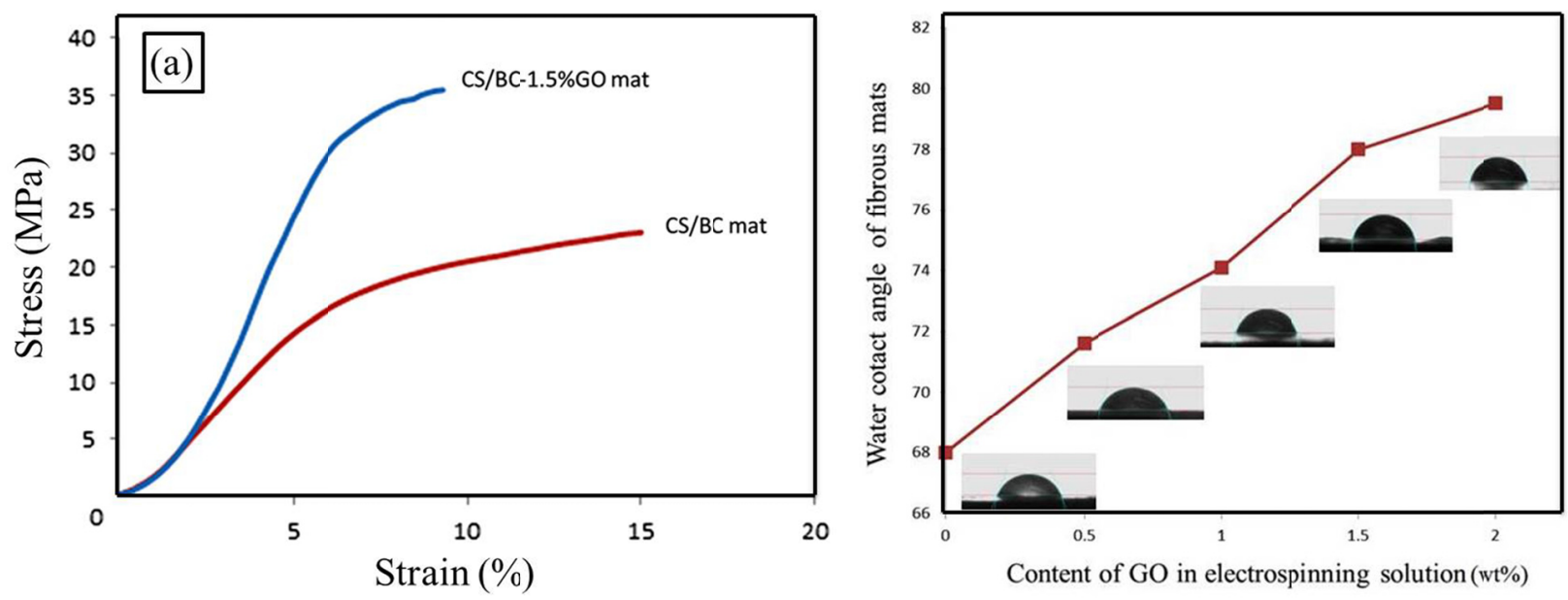
Table(s)

Table 1. Actual levels of design factors.

\begin{tabular}{cccc}
\hline Independent factors & \multicolumn{2}{c}{ Levels } & \\
\cline { 2 - 4 } & -1 & 0 & +1 \\
\hline A: Voltage(kV) & 16 & 19 & 22 \\
B: Distance (mm) & 100 & 120 & 140 \\
C: CS/BC ratio & 1 & 4.5 & 8 \\
\hline
\end{tabular}


Table 2. Box-Behnken experimental design and response results.

\begin{tabular}{cccccc}
\hline $\begin{array}{c}\text { Specimen } \\
\text { number }\end{array}$ & $\begin{array}{c}\text { Voltage } \\
(\mathbf{k V})\end{array}$ & $\begin{array}{c}\text { Distance } \\
(\mathbf{m m})\end{array}$ & $\begin{array}{c}\mathbf{C S} / \mathbf{B C} \\
\text { ratio }\end{array}$ & $\begin{array}{c}\text { Measured fiber } \\
\text { diameter }(\mathbf{n m})\end{array}$ & $\begin{array}{c}\text { Predicted fiber } \\
\text { diameter }(\mathbf{n m})\end{array}$ \\
\hline $\mathbf{1}$ & 16 & 100 & 4.5 & 145 & 166 \\
$\mathbf{2}$ & 22 & 100 & 4.5 & 151 & 140 \\
$\mathbf{3}$ & 16 & 140 & 4.5 & 208 & 210 \\
$\mathbf{4}$ & 22 & 140 & 4.5 & 171 & 184 \\
$\mathbf{5}$ & 16 & 120 & 1 & 217 & 202 \\
$\mathbf{6}$ & 22 & 120 & 1 & 178 & 175 \\
$\mathbf{7}$ & 16 & 120 & 8 & 183 & 148 \\
$\mathbf{8}$ & 22 & 120 & 8 & 147 & 176 \\
$\mathbf{9}$ & 19 & 100 & 1 & 174 & 254 \\
$\mathbf{1 0}$ & 19 & 140 & 1 & 254 & 182 \\
$\mathbf{1 1}$ & 19 & 100 & 8 & 185 & 201 \\
$\mathbf{1 2}$ & 19 & 140 & 8 & 199 & 195 \\
$\mathbf{1 3}$ & 19 & 120 & 4.5 & & \\
\hline
\end{tabular}


Table 3. Analysis of variance (ANOVA) for fiber diameter.

\begin{tabular}{cccccc}
\hline Source & Sum of squares & Degree of freedom & Mean square & F ratio & P value \\
\hline Model & 10867.44 & 9 & 1207.49 & 11.60 & 0.0342 \\
A: Voltage & 1404.50 & 1 & 1404.50 & 13.49 & 0.0349 \\
B: Distance & 3916.12 & 1 & 3916.12 & 37.62 & 0.0087 \\
C: CS/BC ratio & 1485.13 & 1 & 1485.13 & 14.27 & 0.0325 \\
AB & 462.25 & 1 & 462.25 & 4.44 & 0.1257 \\
AC & 2.25 & 1 & 2.25 & 0.022 & 0.8924 \\
BC & 1089.00 & 1 & 1089.00 & 10.46 & 0.0480 \\
$\mathbf{A}^{2}$ & 1316.57 & 1 & 1316.57 & 12.65 & 0.0379 \\
$\mathbf{B}^{2}$ & 11.57 & 1 & 11.57 & 0.11 & 0.7608 \\
$\mathbf{C}^{2}$ & 240.14 & 1 & 240.14 & 2.31 & 0.2261 \\
Residual & 312.25 & 3 & 104.08 & & \\
Correlation total & 11179.69 & 12 & & & \\
\hline
\end{tabular}


Table 4. ANOVA for the adjusted model of fiber diameter.

\begin{tabular}{cccccc}
\hline Source & Sum of squares & Degree of freedom & Mean square & F ratio & P value \\
\hline Model & 10039.24 & 5 & 2007.85 & 12.32 & 0.0023 \\
A: Voltage & 1404.50 & 1 & 1404.50 & 8.62 & 0.0218 \\
B: Distance & 3916.12 & 1 & 3916.12 & 24.04 & 0.0017 \\
C: $\mathbf{C S} / \mathbf{B C}$ ratio & 1485.13 & 1 & 1485.13 & 9.12 & 0.0194 \\
$\mathbf{B C}$ & 1089.00 & 1 & 1089.00 & 6.68 & 0.0362 \\
$\mathbf{A}^{2}$ & 2144.49 & 1 & 2144.49 & 13.16 & 0.0084 \\
Residual & 1140.45 & 7 & 162.92 & & \\
Correlation total & 11179.69 & 12 & & & \\
\hline
\end{tabular}


Table 5. Comparision of mechanical properties of GO modified CS/BC nanocomposites to that of human skin.

\begin{tabular}{ccccc}
\hline Skin location & $\begin{array}{c}\text { Tensile Strength } \\
(\mathbf{M P a})\end{array}$ & $\begin{array}{c}\text { Elastic modulus } \\
(\mathbf{M P a})\end{array}$ & $\begin{array}{c}\text { Elongation at } \\
\text { break (\%) }\end{array}$ & Reference \\
\hline Back & $17.9-36.5$ & $56.8-141.11$ & $20.38-29.52$ & {$[51]$} \\
Back & $13.2-30$ & $48.4-118.2$ & $37-71$ & {$[52]$} \\
Abdomen + Thorax & $2-15$ & 18.8 & & {$[53]$} \\
Forehead and Arm & $5.7-12.6$ & $19.5-87.1$ & $27-59$ & {$[54]$} \\
$\begin{array}{c}\text { CS/BC mat } \\
\text { CS/BC-1.5\%GO } \\
\text { mat }\end{array}$ & $25 \pm 2.1$ & $340 \pm 35$ & $16 \pm 0.3$ & \\
\hline
\end{tabular}

